\title{
SELECTION FOR GAMETE LETHALS AND S-ALLELES IN COMPLEX HETEROZYGOTES
}

\author{
BRIAN CHARLESWORTH \\ School of Biological Sciences, University of Sussex, Brighton BNI $9 Q G$, Sussex, England \\ Received 26.iii.79
}

\section{SUMMARY}

\begin{abstract}
Some hypotheses are proposed for the origin of gamete lethals and $S$-alleles in complex heterozygotes with zygotic lethality. It is shown that egg or pollen detrimentals or lethals are nearly neutral in highly selfing populations, so that a translocation complex which fails to be transmitted through gametes of one sex may be selected if it confers a slight advantage to the sporophyte. Once an asymmetry in transmission of this sort has been established, there is a selective advantage to mutations causing asymmetry in the opposite sense, either by gamete lethality or because of incompatibility (in cases when egg lethality has been established first).
\end{abstract}

\section{INTRODUGTION}

IT is frequently found in Oenothera that the translocation complexes behave as gamete lethals, such that one set of chromosomes (the $\alpha$ complex) is transmitted through the egg only, and the other (the $\beta$ complex) through the pollen only (Cleland, 1972). 'These gamete lethals seem to require a separate explanation from the zygotic lethals discussed in the companion paper to this (de Waal Malefijt and Charlesworth, 1979). Accumulation of deleterious mutations that affect the performance of the gametophyte is less likely than accumulation of recessive or semi-recessive genes affecting the sporophyte, since selection will be more stringent on the former, owing to haploidy. Accumulation is also less likely because the number of loci affecting the sporophyte is probably much larger than the number affecting the gametophyte, so that the total rate of mutation to alleles which are deleterious in the sporophyte is higher than for the gametophyte. Furthermore, the work of Steiner and co-workers on O. biennis I (Steiner, 1956, 1957, 1964; Al Khafaji and Steiner, 1970; Steiner and Levin, 1977) has demonstrated that the apparent pollen lethality of the $\alpha$ complex in this species is due to the fact that the $\alpha$ complexes contain gametophyticallyacting $S$-alleles, such that an $\alpha$ complex containing a given $S$-allele is incapable of fertilising the stigma of a plant carrying the same $S$-allele, but is fully compatible with stigmas of a different type. (The $\beta$ complex behaves as though it contains an allele with no $S$-specificity, i.e. an $S_{f}$ allele). Steiner has proposed that these $S$-alleles were originally present in a self-incompatible ancestral species, from which the $\alpha$ complexes of present-day $O$. biennis 1 were derived by hybridisation with a self-compatible species that donated the $\beta$ complexes. This seems unlikely in view of the fact that the only member of the sub-genus Oenothera known to have an $S$-allele system is $O$. organensis, which is only distantly related to the species of Oenothera that contain translocation complexes (Cleland, 1972, p. 161).

It thus seems more profitable to seek a direct selective explanation of $43 \mid 2-\mathrm{A}$ 
the origin of the gametic lethals and $S$-alleles. At first sight, there is a clear advantage in "efficiency" to a system of balanced gamete lethals, since all the progeny of self-fertilisation are viable compared with one-half with zygotic lethals (e.g. Stebbins, 1971, p. 80). But suitable egg and pollen lethals are unlikely to arise simultaneously in two uniting gametes, so that this advantage is probably a consequence, not a cause. Egg and pollen lethals in separate individuals confer no advantage. It seems more probable that, as pointed out by Cleland (1972, p. 100), the apparent egg lethality of the $\beta$ complexes is simply a consequence of competition between spores as to which forms the embryo sac. The $\alpha$ and $\beta$ complexes of a given strain are known to be highly differentiated genetically, and so it is unlikely that they will be equally fit in the gametophyte stage; any small difference in competitive ability may result in only one complex being successful and hence transmitted through the egg. A similarly competitive situation exists for the growth of pollen tubes down the stigma (Cleland, 1972, p. 103). If the translocation complexes are already behaving as zygotic lethals, it is easily seen that, with complete self-fertilisation, there is no selective disadvantage to a gene which reduces the frequency of transmission of a complex through the ovule if the original complexes are transmitted equally frequently through the pollen. The same is true of a gene which reduces the frequency of transmission of a complex through the pollen, if there is equal transmission through the ovules. Once an asymmetry in transmission frequency has been established for gametophytes of one sex, the presence of zygotic lethals implies a selective advantage to a gene which establishes an asymmetry in the opposite sense in the other sex, since this will increase the frequency of heterozygotes among the progeny. These ideas can be investigated more formally as follows.

\section{Mathematical Models and Results}

\section{(i) Selection on a detrimental gene acting in the egg or pollen}

Assume that we have a population which is segregating for translocation complexes $\alpha$ and $\beta$ that behave as recessive zygotic lethals. All adult plants are therefore $\alpha / \beta$ in constitution. The $\alpha$ and $\beta$ complexes are transmitted with equal frequencies through eggs and pollen. Let the rate of selffertilisation be $s$, such that a fraction $s$ of ovules result from self-pollination and a fraction $1-s$ from random outcrossing. Only 50 per cent of the progeny of each plant are viable $\alpha / \beta$ zygotes. Assume that a mutation arises in the $\beta$ complex to a new state $\beta^{*}$, such that the segregation of $\alpha$ and $\beta^{*}$ in the ovules of $\alpha / \beta^{*}$ plants is modified to $1-k \alpha: k \beta^{*}$. If $\beta^{*}$ carries a detrimental gene affecting the competitive ability of $\beta^{*}$ spores, then $k<\frac{1}{2}$. Let the frequency of $\alpha / \beta^{*}$ adult plants in a given generation be $x$, and that of $\alpha / \beta$ be $1-x$. The values in the next generation, $x^{\prime}$ and $1-x^{\prime}$, are easily seen to be given by the expressions.

$$
\begin{aligned}
& \bar{w} x^{\prime}=\frac{1}{2} s x+\frac{1}{2} k(1-s) x+\frac{1}{2}(1-k)(1-s) x^{2}+\frac{1}{4}(1-s) x(1-x) \\
& \bar{w}\left(1-x^{\prime}\right)=\frac{1}{2} s(1-x)+\frac{1}{4}(1-s)(1-x)+\frac{1}{2}(1-k)(1-s) x(1-x) \\
& +\frac{1}{4}(1-s)(1-x)^{2}
\end{aligned}
$$

where $\bar{w}$ is the mean fitness of the population. 
Writing $y$ for $x /(1-x)$, we obtain

$$
y^{\prime}=y \frac{\left[\frac{1}{2} s+\frac{1}{2} k(1-s)+\frac{1}{2}(1-k)(1-s) x+\frac{1}{4}(1-s)(1-x)\right]}{\left[\frac{1}{2} s+\frac{1}{4}(1-s)+\frac{1}{2}(1-k)(1-s) x+\frac{1}{4}(1-s)(1-x)\right]}
$$

If $s=1$ (complete self-fertilisation), it is obvious from equation (2) that $\beta^{*}$ is completely neutral. If $s<1, \beta^{*}$ is at a selective disadvantage. The dependence of this disadvantage on $s$ can be studied when $\beta^{*}$ is rare, by setting $x=0$ in the factor by which $y$ is multiplied in equation (2), which measures the strength of selection. (It is easily seen that this term is a decreasing function of $x$, so that selection against $\beta^{*}$ is intensified as it becomes more common.) If $k=0$, the strength of selection is equal to $\frac{1}{2}(1+s)$, so that the intensity of selection against a rare egg lethal decreases linearly with the selfing rate.

A similar analysis can be carried out for the case of a mutant complex which is transmitted with lowered efficiency through the pollen. Exactly the same equations result as for an egg detrimental, if $k$ is used to represent the segregation ratio in pollen.

\section{(ii) Selection for a pollen detrimental in the presence of egg lethals or vice-versa}

The above results show that an egg or pollen lethal is at a very small disadvantage in a highly selfing population with zygotic lethals. Any compensating advantages at the other life-cycle stages are quite likely to overcome this disadvantage, leading to the development of lethality in egg or pollen of one of the chromosome complexes. We can then examine the consequences of such lethality for mutations affecting gametes of the opposite sex.

Consider first selection on a mutant $\alpha$ complex, $\alpha^{*}$, when the $\beta$ complex is not transmitted through the egg. $\alpha^{*}$ causes the pollen of $\alpha^{*} / \beta$ plants to segregate as $k \alpha^{*}:(1-k) \beta,\left(k<\frac{1}{2}\right)$. Writing $x$ for the frequency of $\alpha^{*} / \beta$ plants and letting $y=x /(1-x)$, we obtain the expression

$$
y^{\prime}=y \frac{\left[s(1-k)+\frac{1}{2}(1-s)(1-x)+(1-k)(1-s) x\right]}{\left[\frac{1}{2} s+\frac{1}{2}(1-s)(1-x)+(1-k)(1-s) x\right]}
$$

It is easy to see from this equation that, provided $s>0$, the $\alpha^{*}$ complex will replace $\alpha$. When $k=0$ and $\alpha^{*}$ is rare, so that $x$ can be set to zero in the multiplier of $y$ in equation (3), the strength of selection on $\alpha^{*}$ is $1+s$, which increases linearly with $s$.

With $0<s<1$, and $\mathrm{k}=0$ the strength of selection decreases with increasing $x$ to a value of $2 /(2-s)$ at $x=1$. If there were some countervailing disadvantage to $\alpha^{*}$ in the sporophyte stage, such that the fitness of $\alpha^{*} / \beta$ plants was $1-t$ relative to that of $\alpha / \beta$, a polymorphism could be set up, provided $2(1-t) /(2-s)<1<(1+s)(1-t)$. The higher the value of $s$ the less the strength of selection depends on $x$, so that it is unlikely that a pollen lethal could become polymorphic in a highly selfing population.

Exactly similar results can be obtained for the case of a mutation affecting the segregation of the $\beta$ complex in the eggs, when the $\alpha$ complex cannot be transmitted through the pollen. 


\section{(iii) Selection on an incompatibility allele}

It is also of interest to examine the fate of a mutation to an $S$-allele, such that pollen containing the allele has a lowered probability of fertilising the stigma of a plant which also contains it. We can assume without loss of generality that the mutation to this $S$-allele occurs in an $\alpha$ complex, which we can represent as $\alpha_{I}$. Let the frequency of $\alpha_{I} / \beta$ plants be $x$, and write $y=x /(1-x)$. The effect of the $\alpha_{I}$ complex on compatibility can be represented by writing $1-K$ for the probability that an $\alpha_{I}$ pollen grain can fertilise an $\alpha_{I} / \beta$ stigma $(0<K \leqq 1)$; if $K=1$, there is complete incompatibility. The pollen that fertilises the outcrossed ovules of an $\alpha_{I} / \beta$ plant is thus composed of $\alpha_{I}, \alpha$ and $\beta$ in the ratios $\frac{1}{2}(1-K) x: \frac{1}{2}(1-x): \frac{1}{2}$, compared with $\frac{1}{2} x: \frac{1}{2}(1-x): \frac{1}{2}$ for $\alpha / \beta$ stigmas.

Assume first that $\alpha_{I} / \beta$ plants have the same selfing rate, $s$, as $\alpha / \beta$ plants; this is reasonable if $s$ is very close to 1 , so that there is virtual autogamy, as is probably the case in Oenothera (Hoff, 1962). It is easy to derive the following recurrence relation

$$
y^{\prime}=y \frac{\left[\left(1-\frac{1}{2} K x\right)\left\{\frac{1}{2}+\frac{1}{4}(1-s)(1-x)\right\}+\frac{1}{4}(1-s)+\frac{1}{4}(1-K)(1-s) x\right]}{\left[\left(1-\frac{1}{2} K x\right)\left\{\frac{1}{2} s+\frac{1}{4}(1-s)(1-x)+\frac{1}{4}(1-s)\right\}+\frac{1}{4}(1-s) x\right]}
$$

It is easily verified that the terms in square brackets are identical when $x=0$, so that $\alpha_{I}$ is neutral when rare. If $s=1, \alpha_{I}$ remains neutral throughout the frequency range; if $s<1, \alpha_{I}$ becomes selected against as $x$ increases. An $\alpha_{I}$ mutation will only increase in frequency if it confers some other advantage to its carriers; it is least intrinsically disadvantageous with high selfing rates.

It is easy to modify equations (4) to take account of the possibility that $\alpha_{I} / \beta$ have a modified selfing rate, $s_{I}<s$.

We obtain

$$
y^{\prime}=y \frac{\left[\left(1-\frac{1}{2} K x\right)\left\{\frac{1}{2} s_{I}+\frac{1}{4}(1-s)(1-x)\right\}+\frac{1}{4}(1-K)\left(1-s_{I}\right) x\right]}{\left[\left(1-\frac{1}{2} K x\right)\left\{\frac{1}{2} s+\frac{1}{4}(1-s)+\frac{1}{4}(1-s)(1-x)\right\}+\frac{1}{4}\left(1-s_{I}\right) x\right]}
$$

When $x=0$, the strength of selection reduces to $1+\frac{1}{2}\left(s_{I}-s\right)$, so that $\alpha_{I}$ is at a disadvantage even when rare. It can be shown to be disadvantageous throughout the frequency range.

\section{(iv) Selection on an incompatibility allele in the presence of egg lethals}

As in section (ii) above we assume that only the $\alpha$ complex is transmitted through the egg. A mutation to an $\alpha_{I}$ complex of the type described above occurs; the frequency of $\alpha_{I} / \beta$ plants is $x$, and their selfing rate is $s_{I} \leqq s$. Writing $y=x /(1-x)$, we obtain

$$
y^{\prime}=y\left[\frac{s_{I}}{\left(1-\frac{1}{2} K\right)}+\frac{\left(1-s_{I}\right)}{\left(1-\frac{1}{2} K x\right)}\right]
$$

If $K>0, \alpha_{I}$ is clearly at an advantage over $\alpha$, and increasingly so as $x$ increases. Setting $x=0$ and $K=1$ in the bracketed term of equation (6) shows that the strength of selection on a rare gene which confers complete pollen incompatibility is $1+s_{I}$. This may be compared with the corresponding value $1+s$ for a pollen lethal, derived from equation (3). In a 
strongly selfing species with $s \approx s_{I}$, the strength of selection on a rare incompatibility allele is therefore similar to that for a pollen lethal.

\section{Discussion}

It has been shown in section 2 (i) above that a mutation to an egg or pollen detrimental is neutral in a completely selfing population which consists of complex heterozygotes with zygotic lethals. With partial selfing, such mutations suffer a disadvantage in proportion to the outcrossing rate $1-s$. In highly selfing species, a mutation of this sort is liable to become established if it confers a small pleiotropic advantage on the sporophyte, as mentioned in section 2 (ii). It is therefore not difficult to understand how an egg or pollen detrimental or lethal can be established in association with one of the translocation complexes, given the prior existence of zygotic lethals. As shown in section 2 (ii), once such an asymmetry has been established, it creates a selective advantage for an asymmetry in the opposite sense; the establishment of an egg lethal in one complex thus favours the subsequent fixation of a pollen lethal in the other complex and vice-versa, provided there is some degree of self-fertilisation.

We also saw in sections 2 (iii) and 2 (iv) that a mutation causing a selfincompatibility reaction behaves in much the same way as a pollen detrimental. It seems less likely, however, that a gene of this sort will have a pleiotropic favourable effect on the sporophyte, compared with an ordinary egg or pollen lethal, since it is concerned with a rather specialised biochemical function. It is most probable that an $\alpha_{I}$ complex will be selected for after the establishment of an egg lethal or detrimental in the $\beta$ complex. The new $\alpha_{I}$ complex is expected, under these circumstances, to replace the selfcompatible $\alpha$ complex within a local population. Independent mutations to different $S$-alleles in different populations would result in a diversity of $S$-alleles within the same species, and mixing of populations could produce a mixture of $S$-alleles within the same present-day population. Alternatively, if there is some outcrossing, the fixation of one type of $\alpha_{I}$ complex will create a selection pressure for new $\alpha_{I}$ alleles which are self-incompatible but compatible with the established alleles, in a way similar to that for more conventional $S$-allele systems (Wright, 1939). The higher the rate of selffertilisation, the weaker this selection in favour of a diversity of $\alpha_{I}$ alleles. Steiner and Levin (1977) have found high correlations between the type of $S$-allele carried by $\alpha$ complexes in $O$. biennis, and allozyme and morphological markers. This suggests that the diversity of $S$-alleles within a locality is due to the first of the above processes.

The models described above make a number of predictions, which may be compared with observations on Oenothera species:

(a) Zygotic lethality should always precede the evolution of gamete lethals. This entails that zygotic lethals should always be present in populations with gamete lethals, or $S$-alleles, a point which is not easy to establish experimentally. Steiner (1956) has shown that some, at least, of the $\alpha$ complexes of $O$. biennis 1, which contain $S$-alleles, also behave as zygotic lethals. Cleland (1972, p. 29) states that zygotic lethals are possibly present in all lethal bearing races of Oenothera.

(b) Since it is largely a matter of chance which comes first, an egg or 
pollen lethal, it should sometimes be found that a population contains an egg lethal associated with one complex and a zygotic lethal with the other, and sometimes a pollen lethal and a zygotic lethal. Steiner (1956) has demonstrated that this is the case in O. biennis 1 .

(c) There should be a correlation between high selfing rates and the existence of gamete lethals or $S$-alleles. This is more difficult to test in view of the lack of direct estimates of self-fertilisation rates in Oenothera.

(d) $S$-alleles should be found only in association with the $\alpha$ complex, when the $\beta$ complex has a lowered probability of transmission through the egg. This appears to be the case in Oenothera.

\section{References}

AL KhafajI, s. D., AND STEINER, E. 1970. Further analysis of Oenothera biennis populations for incompatibility alleles. Amer. 7. Bot., 57, 183-189.

Cleland, R. E. 1972. Oenothera Cytogenetics and Evolution. Academic Press, London and NeW York.

DE WAAl MALEFIJT, M., AND CHARLESWORTH, B. 1979. A model for the evolution of translocation heterozygosity. Heredity, in press.

HOFF, v. 1962. An analysis of outcrossing in certain complex-heterozygous euoenotheras. I. Frequency of outcrossing. Amer. 7. Bot., 49, 715-724.

stebins, G. L. 1971. Chromosomal Evolution in Higher Plants. Edward Arnold, London. STEINER, E. 1956. New aspects of the balanced lethal mechanism in Oenothera. Genetics, $41,486-500$.

STEINER, E. 1957. Further evidence of an incompatibility allele system in the complexheterozygotes of Oenothera. Amer. 7. Bot., 44, 582-585.

STEINER, E. 1964. Incompatibility studies in Oenothera: the distribution of $S I$ alleles in biennis 1 populations. Evolution, 18, 370-378.

Steiner, E., AND LEVin, D. 1977. Allozyme, SI gene, cytological and morphological polymorphisms in a population of Oenothera biennis. Evolution, 31 127-133.

WRIGHT, s. 1939. The distribution of self sterility alleles in populations. Genetics, 24, $538-552$. 